\title{
Dynamic Scheduling Optimization of Job Shop Based on OCTPN and Hybrid Genetic Algorithms
}

\author{
Xiaoxia Liu" ${ }^{1, a}$, Bingyi Yan ${ }^{1, b}$, Daizhong Bai ${ }^{2, c}$ \\ ${ }^{1}$ Henan University of Technology, Zhengzhou, 450007, China \\ ${ }^{2}$ Heze Power Company Limited, Heze, 274032, China \\ aliuxiaoxia001@163.com, 'bingyi1@163.com, 'baidaizhong@163.com
}

Keywords: Petri net; Hybrid genetic algorithm; Dynamic scheduling; Bi-objective

\begin{abstract}
Combining colored Petri net with object-oriented technology, a new object-oriented colored timed Petri net (OCTPN) method was proposed and the scheduling model of a dual-resource constrained job shop was built. The model has good reusability comparing with the model built by process-oriented technology. A hybrid genetic algorithm based on Pareto was proposed and applied to flexible job shop scheduling problem (FJSP) with bi-objective, where the make-span and the production cost were concerned. The algorithm uses the niche technology and many kinds of crossover operations to get the optimum solutions, most importantly, it can generate new scheduling plan rapidly after the disturbance occurred. The simulation experiment is carried out to illustrate that the proposed method can solve bi-objective job shop scheduling problem effectively.
\end{abstract}

\section{Introduction}

In recent years, some researchers put forward to the scheduling method based on Petri net and do a lot of work on the modeling of flexible job shop using Petri net, but most of them are a process-oriented method, and the model stucture is complex and cannon be reused if the workshop equipment and technology change. In order to reduce model size and increase the reusability of the model, this paper presents a new modeling method, namely OCTPN model.

Job shop scheduling problem is one of the hardest combination optimization problems [1-3]. In recent years, the majority of scholars is to study the single objective scheduling problem, while the study of bi-objective scheduling problems is rarely. In order to solve the bi-objective scheduling problem, this paper put forward a hybrid genetic algorithm based on Pareto.

\section{The OCTPN Model of Job Shop Scheduling}

The modeling of job shop scheduling with OCTPN method has the following steps: (1) Defining the individual objects of system; (2) Finishing the connection between these objects; (3) Determining the color set and initial state of system.

In order to visually explain the OCTPN model of job shop scheduling, a FMS which have ten machines, seven workers and four jobs is studied. Every job has 1-5 procedures. The worker-machine information and the operation time information are shown in Table 1 and Table 2.

Table 1 Worker-machine information

\begin{tabular}{ccccccccccc}
\hline Worker & M1 & M2 & M3 & M4 & M5 & M6 & M7 & M8 & M9 & M10 \\
\hline W1 & $\sqrt{ }$ & $\sqrt{ }$ & - & - & - & - & - & - & - & - \\
W2 & - & $\sqrt{ }$ & $\sqrt{ }$ & - & - & - & - & - & - & - \\
W3 & - & - & $\sqrt{ }$ & $\sqrt{ }$ & - & - & - & - & - & - \\
W4 & - & - & - & - & $\sqrt{ }$ & $\sqrt{ }$ & - & - & - & - \\
W5 & - & - & - & - & - & $\sqrt{ }$ & $\sqrt{ }$ & - & - & - \\
W6 & - & - & - & - & - & - & - & $\sqrt{ }$ & $\sqrt{ }$ & - \\
W7 & - & - & - & - & - & - & - & - & $\sqrt{ }$ & $\sqrt{ }$ \\
\hline
\end{tabular}


Table 2 Operations processing time information

\begin{tabular}{cccccccccccc}
\hline Job & Operation & M1 & M2 & M3 & M4 & M5 & M6 & M7 & M8 & M9 & M10 \\
\hline J1 & $1-1$ & 5 & 7 & - & - & - & - & - & - & - & - \\
& $1-2$ & - & - & 8 & 10 & - & - & - & - & - & - \\
& $1-3$ & - & - & - & - & 5 & 8 & - & - & - & - \\
& $1-4$ & - & - & - & - & - & - & 10 & 11 & - & - \\
& $1-5$ & - & - & - & - & - & - & - & - & 8 & 7 \\
J2 & $2-1$ & 8 & 9 & - & - & - & - & - & - & - & - \\
& $2-2$ & - & - & 10 & 13 & - & - & - & - & - & - \\
& $2-3$ & - & - & - & - & 10 & 12 & - & - & - & - \\
& $2-4$ & - & - & - & - & - & - & - & - & 8 & 7 \\
J3 & $3-1$ & 7 & 9 & - & - & - & - & - & - & - & - \\
& $3-2$ & - & - & 11 & 12 & - & - & - & - & - & - \\
& $3-3$ & - & - & - & - & 8 & 10 & - & - & - & - \\
& $3-4$ & - & - & - & - & - & - & 14 & 13 & - & - \\
& $3-5$ & - & - & - & - & - & - & - & - & 9 & 8 \\
\multirow{2}{*}{ J4 } & $4-1$ & 10 & 13 & - & - & - & - & - & - & - & - \\
& $4-2$ & - & - & 11 & 9 & - & - & - & - & - & - \\
& $4-3$ & - & - & - & - & 10 & 12 & - & - & - & - \\
& $4-4$ & - & - & - & - & - & - & - & - & 8 & 7 \\
\hline
\end{tabular}

At first, we should define the objects in the system. This system has three objects: job object, machine object and worker object. Job object is used to describe system process, called active object, while machine object and worker object are used to support the process, called passive object. Job object only has two states: waiting and being processed. The common activities of machine object instances is that the input buffer of machine gets request from job and is occupied by the job, then sends request to workers to process the job, and the machine release it after the job is processed, then the job enters into the output buffer of machine and is sent out of the output buffer by AGV at last. Worker object has three states: idle, being reserved and operating machines.

Secondly, we finish the connection between three objects. The substructure of job object sends message and requests the input buffer of the machine on which the first procedure of job can be finished when there is a job to wait for being processed. When it is empty, the input buffer will be occupied by the job. Then the substructure of machine object sends message and requests the worker who can operate the machine, if the worker is idle, the job will be processed. The worker becomes idle again after the job is finished, the substructure of machine object will reply message to the substructure of job object and the substructure of job object will send new message and request the input buffer of the machine on which the next procedure of job can be finished, this cycle will continue until all jobs are finished. The model is shown in Fig.1.

At last, we should identify the color set and initial state of system. The color set of job object is as follows: $C_{0}=\left\{C_{0}^{1}, C_{0}^{2}, C_{0}^{3}, C_{0}^{4}\right\}, C_{0}^{1}=\left(J_{1}, 1,\left(M_{1} / M_{2} M_{3} / M_{4} M_{5} / M_{6} M_{7} / M_{8} M_{9} / M_{10}\right),(5 / 7,8 / 10,5 / 8,10 / 11,8 / 7)\right)$. The color set of machine object is as follows: $C_{1}=\left\{C_{1}^{1}, C_{1}^{2}, \cdots, C_{1}^{10}\right\}, C_{1}^{1}=\left(M_{1}, 1, W_{1}\right)$. The color set of worker object is as follows: $C_{2}=\left\{C_{2}^{1}, C_{2}^{2}, \cdots, C_{2}^{7}\right\}$, here $C_{2}^{1}=\left(W_{1}, 1, \varnothing\right)$. The initial state of system is below: $M_{0}\left(P_{1}^{1}\right)=\left\{C_{0}^{1}, C_{0}^{2}, C_{0}^{3}, C_{0}^{4}\right\} ; M_{0}\left(P_{2}^{1}\right)=M_{0}\left(P_{2}^{8}\right)=M_{0}\left(P_{2}^{9}\right)=\left\{C_{1}^{1}, C_{1}^{2}, \cdots, C_{1}^{10}\right\} ; M_{0}\left(P_{3}^{1}\right)=\left\{C_{2}^{1}, C_{2}^{2}, \cdots, C_{2}^{7}\right\}$.

Here, $p_{1}^{1}$ and $p_{1}^{2}$ is the two states of job; $p_{2}^{1}$ : the input buffer is empty; $p_{2}^{2}$ : the input buffer is being reserved; $p_{2}^{3}$ : the input buffer is being occupied; $p_{2}^{4}$ : machine is being occupied; $p_{2}^{5}$ : machine is running; $p_{2}^{6}$ : the job is waiting for being sent to the output buffer; $p_{2}^{7}$ : the output buffer is occupied; $p_{2}^{8}$ : the machine is idle; $p_{2}^{9}$ : the output buffer is idle; $p_{2}^{10}$ : the machine breakdown maintenance; $p_{3}^{1}, p_{3}^{2}$ and $p_{3}^{3}$ is the three states of worker; $t_{1}^{1}$ : the job applies for being processed; $t_{1}^{2}:$ the next procedure of job is bing processed; $t_{2}^{1}:$ the input buffer is reserved; $t_{2}^{2}:$ the input buffer is occupied; $t_{2}^{3}:$ the machine is reserved; $t_{2}^{4}$ : the machine is machining the job; $t_{2}^{5}$ : the machine finishs the job; $t_{2}^{6}:$ the output buffer is 
occupied; $t_{2}^{7}$ : the output buffer is occupied; $t_{2}^{8}$ : the machine breaks down; $t_{2}^{9}$ : the machine is repaired; $t_{3}^{1}$ : the worker is reserved; $t_{3}^{2}:$ the worker operaters the machine; $t_{3}^{3}:$ the worker finishs operation; $m_{1}$ : applying for the machine; $m_{2}$ : applying for the next procedure; $m_{3}:$ applying for the worker; $m_{4}:$ the worker applies for operating; $m_{5}$ : the machine finishs machining and releases the worker; $m_{6}$ : applying for AGV to convey the job.

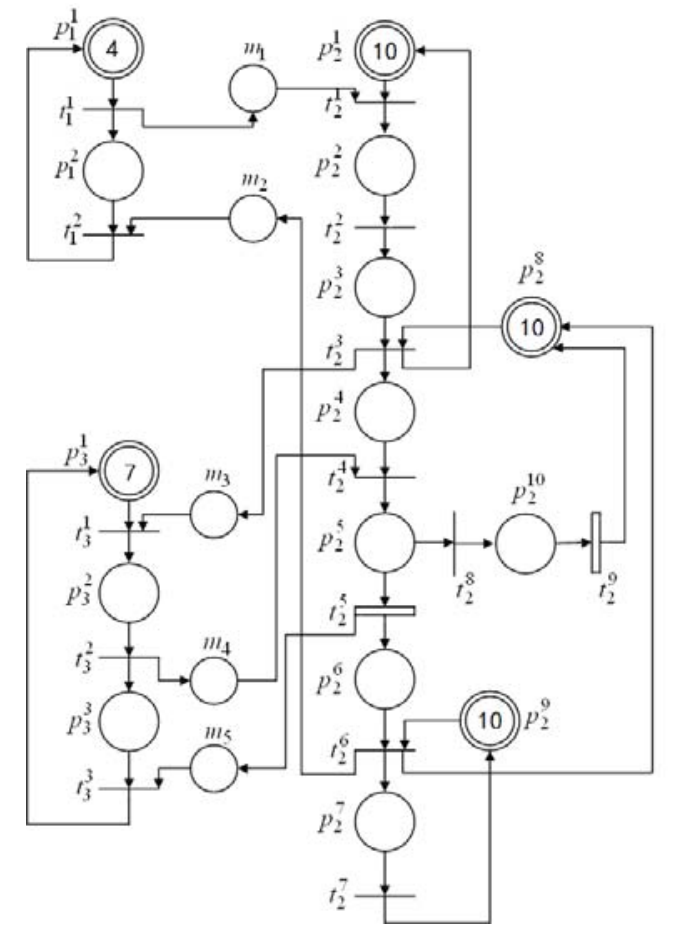

Fig.1 The OCTPN model of job shop sheduling

\section{Hybrid Genetic Algorithm}

Objective Function. The paper proposes a compositive objective of the job shop scheduling problem: Shortening the makespan as soon as possible to improve work efficiency; Reducing the production cost as much as possible to save the cost.

Gene Encoding and Decoding. Operation-based representation is adopted. The optimum solution exists in active schedule, so the decoding method will generate active schedule.

Pareto-ranking Approach. All Pareto optimal individuals in current population are ranked by 0, then delete them from the population, all Pareto optimal ones in remaining population are ranked 1, and so on, until all individuals are determined.

Niche Technology. The niche technology has been devised to prevent genetic drift.

Crossover and Mutation. MPPX, MGOX, MGPMX1 and MGPMX2 [4] and SWAP mutation method are adopted to inherit excellent modes of parents.

\section{Discussion and Conclusion}

The algorithm parameters are that population size is 300 , crossover rate is 0.8 , mutation rate is 0.01. Some of the initial parameter values are seen in the literature [5].

If it broke down at time 20, the machine 5 cannot participate in the rest scheduling task, and the optimum scheduling result (60 and 6858.15) is showed in Fig.2.

The Gantt charts present the relation of machines/workers and operations. In the Gantt chart of machines and operations, the $\mathrm{X}$-coordinate denotes the processing time of jobs, and the $\mathrm{Y}$-coordinate denotes machine number. Every operation is expressed using a rectangle and a four-figure number. The first two numbers denote job number, the third is operation number, and the last one presents the worker who finishes this operation. For example, the first task on Machine 1 is "0311" in Fig.2, it 
means that the operation 1 of job 3 is done by the worker 1 . In the Gantt chart of workers and operations, the Y-coordinate denotes worker number. Every operation is expressed with a rectangle and a four/five-figure number. The first three numbers are the same as above, and the rest is the machine number which is used to process this operation. For example, the first task of worker 2 is "0112", it means that the task 1 of job 1 is done by the machine 2 .

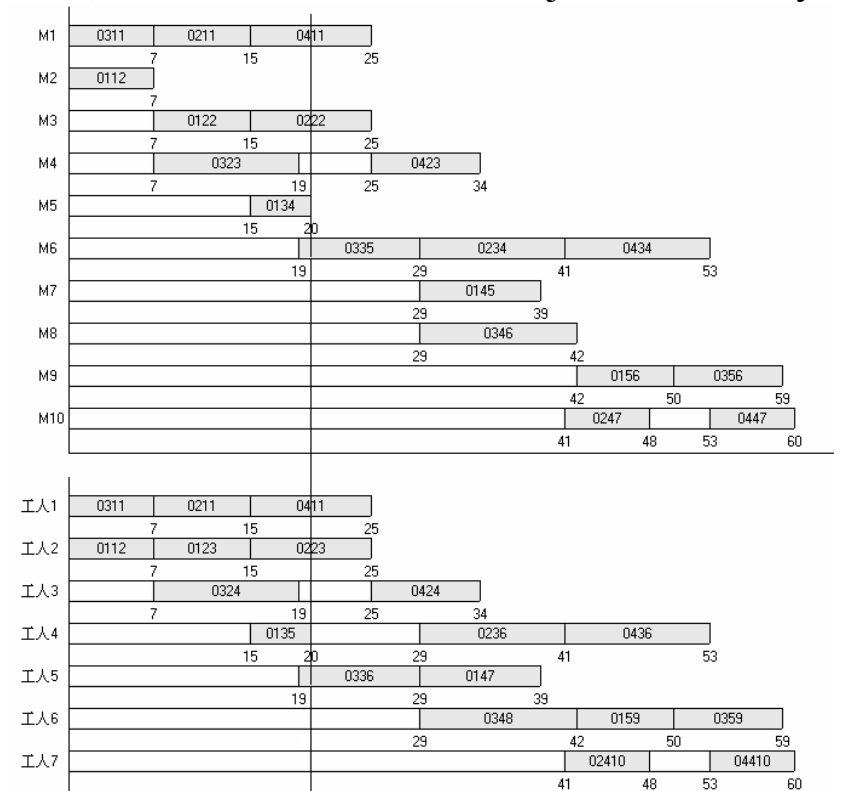

Fig. 2 Gannt graph after machine 5 in big trouble

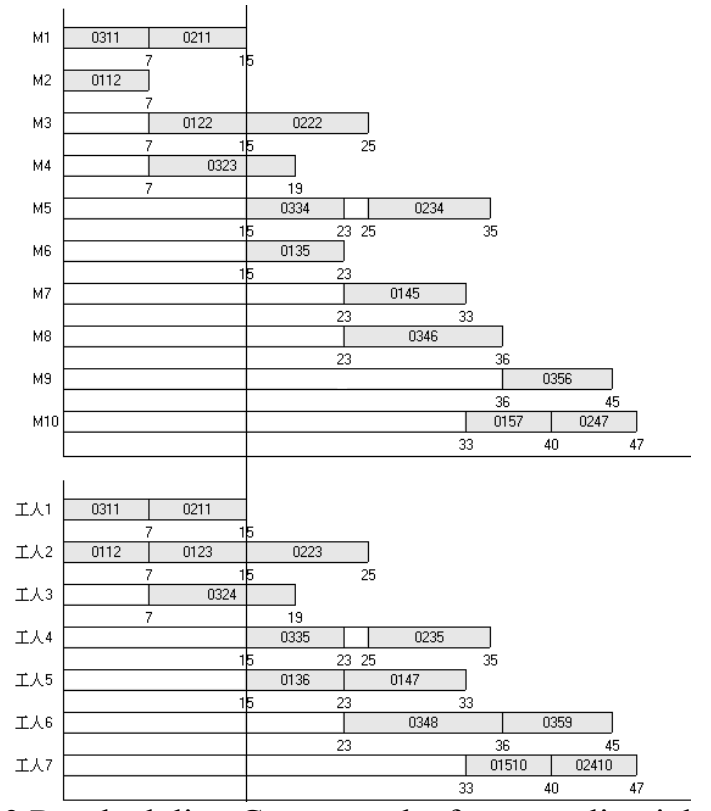

Fig.3 Rescheduling Gannt graph after canceling job 4

If job 4 was cancelled at time 15, the rest tasks need to reschedule because it may cause some workers and machines have a longer leisure time and reduce the work efficiency of machines and workers. The optimum scheduling result (47 and 5402.59) is showed in Fig.3.

The OCTPN model presented in this paper obviously reduces model size and increases the reusability of the model. The hybrid genetic algorithm developed in this paper is proved to be very useful for optimizing the production performance under the realistic conditions.

\section{Acknowledgements}

This work was financially supported by the Education Department of Henan Province Natural Science Research Project (2010B460003), Henan University of Technology Doctoral Fund (150348).

\section{References}

[1] W.L. Wang, L. Wang. Dynamic Job Shop scheduling based on hybrid differential evolution algorithm. Computer Integrated Manufacturing Systems, vol.18(2012), p. 531-539, in Chinese.

[2]J.Y.Gao, P. Zhang, H.F. Yu. A survey on job shop dynamic scheduling. Electrical and Mechanical Engineering Technology, vol.38(2009), p.13-16, in Chinese.

[3]P. Xu, Y.Wang. Research and application of shop dynamic scheduling algorithm. Aeronautical Manufacturing Technology, vol.21(2009), p.82-87, in Chinese.

[4] Byung J, Hyung R, Hyun S: A hybrid genetic algorithm for the job shop scheduling problems. Computers \& Industrial Engineering, vol. 45(2003), p. 597-613.

[5] X. X. Liu, L. Y. Xie, Z. Tao: Flexible Job Shop Scheduling for Decreasing Production Costs. Journal of Northeastern University (Natural Science), vol. 29(2008), p. 561-564, in Chinese. 